\title{
XIX. Abstract of the principal demonstrations of M. Fourier, relative to the mathematical law of the radiation of heat. Communicated in a Letter to Sir Davis Brewster
}

\section{Baron Maurice}

To cite this article: Baron Maurice (1833) XIX. Abstract of the principal demonstrations of $\mathrm{M}$. Fourier, relative to the mathematical law of the radiation of heat. Communicated in a Letter to Sir Davis Brewster, Philosophical Magazine Series 3, 2:8, 103-110, DOI: 10.1080/14786443308647982

To link to this article: http://dx.doi.org/10.1080/14786443308647982

曲 Published online: 01 Jun 2009.

Submit your article to this journal $₫$

Џll Article views: 2

Q View related articles ¿ 
and again from the 4th to the 12th of April; the remainder, however, of this month was for the most part gloomy and exceedingly cold, and on the 25th the summit of Cross Fell was pretty thickly covered with snow. During the first three weeks of May, keen cutting winds prevailed generally from the East; on the 2nd, there was snow several inches in depth in the vicinity of Tindal Fell, and on the 15 th and 16th we had several smart hail-showers; in short, it was not before the 22nd that the weather became at all warm and seasonable.

Vegetation, as might naturally be expected, made but little progress; and upon the whole the spring of this year was perhaps more backward than the very late one of 1829.

The summer and autumn which followed, however, were fine and remarkably dry, the harvest early, and the crops in this district, generally speaking, were exceedingly good, more particularly on cold elevated grounds, where the farming produce was scarcely ever recollected to have been more abundant, or to have been secured in finer condition.

Carlisle, November 10, 1832.

XIX. Abstract of the principal Demonstrations of M. Fourier, relative to the Mathematical Law of the Radiation of Heat. By Baron MAURICE, Member of the Institute of France, and Professor of Analytical Mechanics in the Academy of Geneva. Translated by James D. Forbes, Esq. F.R.S. L. \& Ed. F.G.S.Sc. Communicated in a Letter to Sir David Brewster.

My Dear Sir,

THE précis of Fourier's Demonstrations, from which the following translation is taken, was put into my hands in manuscript, by my friend Baron Maurice of Geneva, last month. It was written for the Supplement to M. Prevost's work on Heat, which has since appeared. As I have not observed any account of this part of Fourier's labours in English works (and, indeed, the writings of that distinguished man are too little known in this country), I think the present notice may not be unacceptable; more especially as the original Memoir of Fourier, in the fifth volume of the Memoirs of the Institute, is really obscure. In this tract M. Maurice has reduced the theory to a few simple propositions, which he has given with all that copiousness of reasoning which distinguishes the writings of Fourier, when he is establishing fundamental propositions upon which a complex superstructure is to be raised : the first demonstration in particular is quite in the Newtonian style. I have adhered closely to the 
104. M. Maurice's Abstract of Fourier's Demonstrations

text of M. Maurice, though I have not always translated it word for word.

I am, my Dear Sir, yours most faithfully,

Greenhill, Edinburgh, Dec. ơth, 1832.

JaMes D. Forbes.

Abstract, \&c.

1. Law of Radiation.- "The rays of heat which issue under different angles from the same point of the surface of any body, have an intensity which decreases proportionally to the sine of the angle formed by their direction with the plane tangential to the surface, at the point of emission."

Demonstration. - Let AB, (fig. 1.) be the mathematical surface of the body; and AC the thickness of its physical surface. We shall consider a normal ray of heat $\mathrm{CA}$, and conceive that $C$ is the point furthest from the surface capable of emitting any heat whatever by radiation. Consequently, as weadvance from $C$ to $A$, the particles radiate heat more copiously.

Fig. 1.

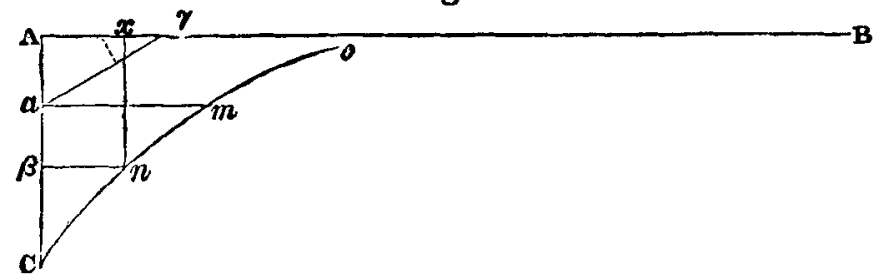

Fig. 2.

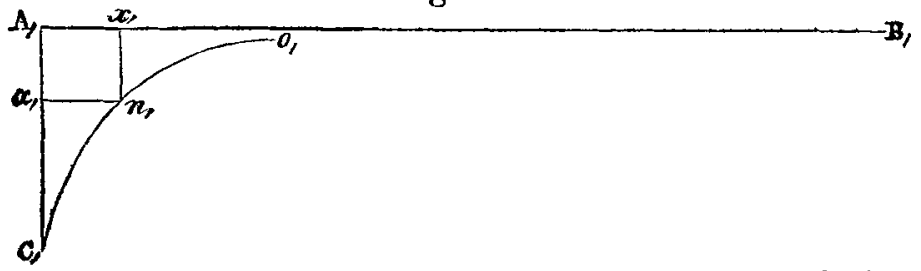

Let us assume the quantity of heat to be known which is furnished by each point $\alpha \beta$.......situated in the normal direction, and that it is represented by the respective abscissa $\beta n$ a $m$.... referred to the axis $\mathrm{A} B$ : we may conceive such a curve as $\mathrm{C} n m o \ldots$ passing through their extremities; and the sum of these abscisse, or the area of the curve, will express the intensity of the ray of heat normal to the surface.

We must next consider the intensity of a similar ray in the direction $\alpha \gamma$, inclined to the mathematical surface at an angle $\phi$. Let us consider first the point $\alpha$ of the normal ray. 
The quantity of heát which reaches $\gamma$ from $\alpha$ will be identical with that which reaches $A$ from $\beta$, provided $A \beta=\alpha \gamma$. Now, by the hypothesis, the abscissa $\beta n$ represents this quantity. Next let us take (fig. 2.) $A_{l}, B_{l}, C_{l}$ and $\alpha_{l}$ as before, and erect the abscissa $\alpha_{1} n$, equal and parallel to $\beta n$, and let us repeat a similar operation for all the other points of the line $\alpha \gamma$; we shall then have another curve $C_{1} n_{l} o_{l} \ldots$ of which the surface will represent the intensity of a ray inclined to the normal.

In order to compare the surfaces of the curves fig. 1 and 2; we have only to observe, that for the same abscissa $\mathrm{A} x=\beta n$ (fig. 1.), or $\mathrm{A}_{1} x_{1}=\alpha_{1} n_{1}$ (fig. 2.), if the ordinate (fig. 1.) is represented by $A \beta=\alpha \gamma$, and we make $\alpha \gamma=1$, the ordinate $A, \alpha$, (fig. 2.) equal to $A \alpha$ will be represented by $\sin \varphi$. But when two curves have the same origin and axis of abscissæ, their surfaces comprised between the origin and a common limit are evidently in the relation of the respective ordinates: therefore, the surface $\mathrm{AC} n o:$ surface $\mathrm{A}, \mathrm{C}, n, o,=1: \sin \varphi$. Wherefore the respective intensities of a ray of beat $\mu$ normal to the surface, and one, $\nu$ inclined at an angle $\varphi$ have the same ratio, and $\nu=\mu \sin \varphi$. Q. E. D.

2. This demonstration is equally applicable to curve as to plane surfaces. For the thickness of the physical surface CA being extremely small, the portion of the mathematical surface included between the extremity of the normal and that of the oblique line (which at most can only be equal to the length of CA), will always be sufficiently small to be confounded with a plane tangential to the point of emission.

3. The absolute intensities which are supposed to be known in the preceding demonstration are in no respect wanted for the determination of the intensity of the oblique rays relatively to the normal ray.

4. Having demonstrated the law of radiation upon these simple principles, we proceed to show, that did this law not exist we should arrive at conclusions at variance with the simplest experiments. But we must first introduce a distinct conception of the radiating power of a given surface.

5. Let $a$ be the temperature of a heated surface, and $h$ its radiating power*. Each infinitely small portion of the surface may be viewed as the centre of a hemisphere which is filled by the radiant heat emanating from it. If then we consider a small portion of the surface taken as unity, the quan-

- This coefficient $h$ depends on the nature of the radiating body, and is what Fourier calls "Conducibilité extérieure" in his Traité Analytique de Chaleur.-TransLator,

Third Series. Vol. 2. No. 8. Feb. 1833. 


\section{6 \\ M. Maurice's Abstract of Fourier's Demonstration}

tity of heat radiated by it will be proportional to the product $a h$; and if we could know how much heat traversed in unity of time the surface of a hemisphere of radius 1, having for its centre an element of surface taken for' unity, we should have the value of $h$ by dividing the expression for that quantity by the product $a .2 \pi$.

In order to determine this quantity, let us designate by $g$ the constant coefficient which represents the intensity of a ray of heat normal to the surface. If this intensity varies with the inclination $\phi$ of the rays, we may represent it by $g f(\varphi)$, where $f(\varphi)$ denotes an unknown function of the inclination. Hence $a g f(\phi)$ will represent the heat afforded by a ray making an angle $\varphi$ with the surface.

Let us next consider upon the hemisphere of radius 1 , an elementary zone which has for height the element $d \phi$ of the $\operatorname{arc} \varphi$, and for base the circle $2 \pi \cdot \cos \varphi:$ it is evident that the product $a g \cdot f(\phi) \cdot 2 \pi \cos \phi d \phi$ will express the quantity of heat which in unity of time will traverse the surface of the elementary zone; and consequently the integral of this expression taken from $\phi=0$ to $\phi=\frac{1}{2} \pi$, will express the quantity of heat which proceeding from unity of surface will traverse in unity of time the hemispherical surface $2 \pi$. But this quantity ought also to be exactly represented by $a h 2 \pi$.

$$
\text { Hence } \quad 2 a \pi \cdot h=2 a \pi \cdot g f d \varphi \cos \varphi \cdot f(\varphi)
$$

or, more simply, $\quad h=g \int d \phi \cos \varphi \cdot f(\varphi)$,

taking the integral between the limits first assigned. Such will be the general expression of the radiating power of a given surface.

Thus, for example, if the intensity of the rays be the same for all angles of inclination, we have $f(\varphi)=1$, and integrating the expression for $h$ between the given limits, we have $h=g$, as it ought to be upon this supposition.

If, on the contrary, as we have seen in article 1, the intensity is proportional to the sine of the angle of emission, we shall have $f(\phi)=\sin \phi$, which gives $h=\frac{1}{2} g$. Hence in the case of nature, in which the general intensity of the rays is expressed by $g . \sin \varphi$, it has for extreme values zero and $g$; and the mean value of $h$, the radiating power, is $\frac{1}{2} g$. Such would be the intensity of rays emitted at an angle of $30^{\circ}$, for,

$$
g \cdot \sin \frac{\pi}{6}=\frac{1}{2} g .
$$

We also see that if all the rays were similar to those nor- 
mal to the surface, the resulting effect would be double what it really is; for it would then be represented by $a g$ instead of $\frac{1}{2} a g$ or $a h$.

6. From these principles may be deduced some curious and important consequences : but we proceed at present to consider a particular case, which puts in a strong point of view the necessity of the law of the sines.

7. Let us inquire what would be the final temperature acquired by a spherical molecule placed in the centre of a spherical surface having a radius $R$, which we conceive to be constantly kept at the temperature $a$; and continuing to call $h$ the radiating power of the surface both of the spherical inclosure and of the molecule, of which we may call the radius $r$, we shall have, as we have just seen,

$$
h=g \int d \phi \cos \phi \cdot f(\phi) \ldots \ldots \ldots
$$

denoting by $\phi$ the inclination of the rays as before.

Let $\omega$ be an infinitely small portion of the interior spherical surface. It will constantly emit rays of heat which may be conceived in unity of time to fill a hemisphere having a radius $R$ : now the rays normal to the interior spherical surface will necessarily fall upon the central molecule, and will occupy upon the surface $2 \pi . R^{2}$ of the hemisphere a space equal to $\pi r^{2}$. Hence these normal rays, all which have the intensity $g$, and of which $\omega$ is the base, will transmit to the central molecule a quantity of heat expressed by

$$
\omega . a g \cdot \frac{\pi r^{2}}{2 \pi \mathrm{R}^{2}} \ldots \ldots \ldots .
$$

If in this expression we put for $g$ its value found by equation $(\alpha)$, it becomes

$$
\omega \cdot \frac{a r^{2}}{2 \mathrm{R}^{2}} \cdot \frac{h}{f d \phi \cos \varphi \cdot f(\phi)} \ldots \ldots \ldots
$$

and as the ratio of the whole spherical surface to $\omega$ is expressed by $\frac{4 \pi \mathrm{R}^{\mathrm{a}}}{\omega}$, if we multiply this ratio by the expression (2), we shall have the whole quantity of heat received by the molecule, denoted by $2 \pi \cdot a r^{2} \cdot \frac{h}{\int d \phi \cos \phi \cdot f(\phi)}$, the limits of the integral being always 0 and $\frac{1}{2} \pi$.

Let us now suppose that the final temperature acquired by the molecule is represented by $b$; it follows that the molecule will dissipate from its surface a quantity of heat equal to 
$4 \pi r^{3} . b h$. We shall then have, for unity of time, the equation

or, simplifying,

$$
4 \pi r^{2} \cdot b h=2 \pi r^{2} \cdot a h \cdot \frac{1}{\int d \varphi \cdot \cos \varphi \cdot f(\varphi)},
$$

$$
b=\frac{1}{2} a \cdot \frac{1}{f d \varphi \cos \varphi \cdot f(\varphi)} .
$$

But if the intensity of the rays does not vary with their inclination, we shall have $f(\phi)=1$, and taking the integral between the proper limits, $b=\frac{1}{2} a$; so that the central molecule could only acquire a temperature equal to half that of the spherical inclosure ! - a result which is absurd, being constantly contradicted by experience. If on the contrary we make $f(\phi)=\sin \phi$, we find rigorously $b=a$; that is, that the final temperature of the molecule is equal to that of the inclosure,-agreeably to experiment.

8. It is easy to explain the rather singular result at which we have just arrived; namely, that if the intensity of the rays of heat emitted were independent of the angle of emission, the central molecule would only acquire half the temperature of the inclosure in which it is placed, even after an indefinite time. For whilst the inclosure from its spherical form can only transmit to the central molecule such rays as are normal to its own surface, its calorific energy being thus independent of the angle of emission of the other rays, the molecule itself dissipates heat in all directions, and (according to the hypothesis) with equal intensity; it is evident (see art. 5.), from the equation $h=g$, which is then applicable, that it will lose in unity of time twice the quantity of heat which it receives; its temperature therefore will only be half that of the inclosure*.

9. We shall next proceed to show the necessity of the mathematical law of radiation by proving that its existence is essential, in order to account for the uniformity of temperature pervading a space of which the limits are kept during a sufficient time at a constant temperature,-a fact which experience demonstrates.

10. Let us consider in the interior of the bounding sides

* These facts tend also to establish the rigorous connexion betweeu the absorptive and emissive powers of bodies for radiant heat. If in the experinent ahove described, the central molecule had one of these properties in the slightest degree in excess over the other, it might acquire an infinitely high or an infinitely low temperature. Thus by these elementary views the necessity of these two fundamental laws diseovered experimentally by Professor Leslie is illustrated.-Translation. 
of the Mathematical Lare of the Radiation of Heat. 109

of the given space, which have the common temperature $a$, two extremely small portions of the surface, which are plane and homogeneous, and which may be denoted by $s$ and $s$. Let $\delta$ be the distance between $s$ and $s^{\prime}$, which is finite, and therefore incomparably greater than the dimensions of these very small portions of surface:-we have to find how much heat the surface $s$, for example, receives from $s$, in unity of time; neglecting, as we have hitherto done, the portion of heat reflected, since we shall do the same when we come to consider how much heat $s$ receives from $s^{\prime}$.

Let us call $p$ the angle which $\delta$ makes with $s$, and $\phi$ that which it makes with $s^{\prime}$. We may reckon the distance $\delta$ from any points of the surfaces $s$ and $s$, since from their small size no sensible variation could be introduced into the length of $\delta$, or the angles $p$ and . Each infinitely small portion $\omega$ of the surface $s$ will be the base of a ray of heat falling upon $s^{\prime}$; and if, to know how much heat this ray contains, we make through a point of $s^{\prime}$ a section of the ray perpendicular to its direction, we shall obviously have $s^{\prime} \sin \phi$ for the area of that section; a quantity of which we must take the ratio to the whole surface $2 \pi \delta^{2}$ of the hemisphere traversed by all the rays emanating from $\omega$, when we wish to measure the quantity of heat which falls from $s$ upon $s$.

Now, denoting by $f(p)$ the unknown function of the inclination $p$ of the ray which determines its intensity, we shall have the product $w . a g \cdot f(p)$ for the heat of the pencil emitted from $\omega$ at an inclination $p$; $g$ representing, as before, the intensity of a ray normal to the surface. Then multiplying this by $\frac{s}{\omega}$, and also by $\frac{s^{\prime} \sin \varphi}{2 \pi \delta^{23}}$, the ratio of the surfaces, we have the expression $\frac{a g}{2 \pi \delta^{3}} \cdot s f(p) \cdot s^{\prime} \sin \varphi$

for the total amount of heat passing from the surface $s$ to the surface $s^{\prime}$.

But it is evident that, reasoning in a similar manner, the quantity of heat passing from $s^{\prime}$ to $s$ in the same unity of time will be

$$
\frac{a g}{2 \pi \delta^{2}} \cdot s^{\prime} f(\varphi) \cdot s \sin p \text {. }
$$

It follows from the comparison of these expressions, that if the unknown function $f(p)$ or $f(\varphi)$ be the sine of those angles, the action of $s$ will be the same upon $s^{\prime}$ as that of $s^{\prime}$ is upon $s$; and that if this function does not represent the sine, these two actions cannot be equal.

Hence it is easy to see that unless this condition be ful- 
110 Sir J. F. W. Herschel's Notice of a remarkable Deposition

filled, an equilibrium of temperature cannot be established after any lapse of time.

11. Corollary.-Since (art. 5.) we have found $g=2 h$ in the case of nature, we shall have for the expression of $s$ upon $s^{\prime}$, or of $s^{\prime}$ upon $s$,

$$
\frac{a h}{\pi} \cdot \frac{s \sin p \cdot s^{\prime} \sin \varphi}{\delta^{2}} .
$$

But if the surfaces have unequal temperatures $a$ and $b$, and supposing, for example, that $a$ is greater than $b$, the result of their mutual action will be proportional to

conformably to experience.

$$
(a-b) h \cdot \frac{s \sin p \cdot s^{\prime} \sin \varphi}{\delta^{2}}
$$

XX. Notice of a remarkable Deposition of Ice round the decaying Stems of Vegetables during Frost. By Sir JoHN F. W. HerscheL, K.H. F.R.S. \&c.\&c.

[With Figures : Plate II.]

To R. Phillips, Esq. \&c. \&c.

Dear Sir,

SOME years ago during the first days of a sharp frost, my attention was attracted by the unusual accumulation of ice round the roots and stumps of some dry and decaying thistles in the fields; while at the same time comparatively little hoarfrost was deposited on wheat-stubble and other vegetables in the neighbourhood. On examination I found it to incrust the stalks in a singular manner in voluminous friable masses, which looked as if they had been squeezed, while soft, through cracks in the stems. It was chiefly or entirely confined to the immediate neighbourhood of the root, the upper parts of the taller unbroken stalks being quite free from it. This peculiarity of situation, and the comparative absence of hoarfrost elsewhere, induced me at the time to attribute it either to some different cause from hoar-frost, or to some singular modification of that atmospheric deposition by local and temporary circumstances.

The above observation was recalled to my recollection by a similar phænomenon noticed on the morning of the 11 th instant, after a night of sharp frost, of the kind vulgarly called " a black frost ;" there being little deposition of hoar-frost from the air, which was during the night extremely tranquil, with a scarcely perceptible current from the north-east. The 